\title{
From Obesity to Chronic Kidney Disease: How Can Adipose Tissue Affect Renal Function?
}

\author{
Marina Martin-Taboada Rocío Vila-Bedmar Gema Medina-Gómez \\ Departamento de Ciencias Básicas de la Salud, Área de Bioquímica y Biología Molecular, Facultad de Ciencias de la \\ Salud, Universidad Rey Juan Carlos, Madrid, Spain
}

\section{Keywords}

Obesity · Adipokines · Chronic kidney disease · Bariatric surgery $\cdot$ MicroRNAs

\begin{abstract}
Obesity is directly associated with an increased risk of developing CKD, regardless of other comorbid conditions. Although the molecular mechanisms that link both diseases are not well established, the role of adipose tissue (AT) is becoming increasingly important in obesity-associated kidney damage. In the context of obesity, lipotoxicity and the alteration of AT secretion profile promote inflammation, oxidative stress, and fibrosis in the kidney, which ultimately leads to impaired renal function. Different studies have highlighted the importance of body weight loss in the improvement of renal function markers. In this regard, bariatric surgery, rather than low-calorie diets, has been accepted as the most effective option to lose weight. In fact, a significant reduction in proteinuria and hyperfiltration has been observed in association with surgically induced weight loss. Detection of early signs of kidney dysfunction in patients with obesity has not been accomplished yet, though. Therefore, understanding the harmful effects within the adipo-renal axis is essential to prevent the progression to the irreversible renal
\end{abstract}

insufficiency. MicroRNAs have recently been described as important modulators of normal kidney function. Some of these microRNAs could be potential early markers of kidney damage, which would help with the diagnosis and the prevention of CKD.

(c) 2021 S. Karger AG, Basel

\section{Introduction}

CKD represents one of the most under-recognised diseases worldwide. The increase in the prevalence of CKD has been accompanied by a significant rise in obesity. Indeed, obesity not only increases the prevalence of type 2 diabetes (T2D) and hypertension, both important risk factors for CKD, but also predicts an increased incidence for the development of CKD and its progression to ESRD per se [1]. However, how obesity impairs kidney function remains poorly understood. In this minireview, we describe different mechanisms underlying obesity-related CKD that may aid the identification of new biomarkers and discuss the current knowledge in this field.

Contribution from the CME course of the DIABESITY Working Group of the ERA-EDTA, Alcorcón (Madrid), Spain, November 15-16, 2019. karger@karger.com

www.karger.com/nef

(c) 2021 S. Karger AG, Base

Karger ${ }^{\prime}=$
Correspondence to:

Rocío Vila-Bedmar, rocio.vila@urjc.es

Gema Medina-Gómez, gema.medina@urjc.es 


\section{Obesity-Related CKD}

Renal effects of obesity are both structural and functional. The study of patients with different degrees of obesity has led to the designation of an obesity-related glomerulopathy (ORG), where glomerular hypertrophy seems the initial lesion stimulating podocyte effacement and triggering the inflammatory response (reviewed in [2]). Pro-fibrogenic signals induce the deposition of the extracellular matrix in the nephrons, leading to basement membrane thickening, glomerulosclerosis, and tubulointerstitial fibrosis. Since podocytes cannot cover the expanded glomerular surface, this leads to podocyte dysfunction and effacement; the glomerular filtration barrier breaks, and the remaining glomeruli overstrain to compensate it, eventually giving rise to hyperfiltration and proteinuria [2]. However, not all individuals with obesity develop CKD, since the so-called metabolically healthy obese do not present an increased risk for the development of CKD [3], suggesting that an increased BMI is not enough to induce kidney damage.

\section{Involvement of Adipose Tissue in Obesity-Related CKD}

\section{Adipose Tissue-Derived Lipotoxicity}

In obesity, the energy excess leads to a chronic stressful environment, resulting in adipose tissue (AT) hypertrophy until adipocytes reach their expansion limit. From this point on, the excess of toxic lipid species accumulates ectopically in different organs, inducing a harmful effect known as lipotoxicity. Especially in the kidney, this is associated with structural and functional changes of mesangial cells, podocytes, and proximal tubular cells [4]. In podocytes, this would interfere with the insulin pathway, critical for survival and maintenance of the podocyte structure, leading to podocyte apoptosis [5] and inducing a compensatory hypertrophic response in the remaining podocytes [2].

\section{Alterations in the AT Secretion Pattern}

$\mathrm{AT}$ is an important source of active protein factors, known as adipocytokines, which participate in different metabolic processes [4]. Alterations in the secretion and signalling of AT-derived molecules during obesity largely mediate the pathogenesis of metabolic disorders. Indeed, obesity is characterized by an increased secretion of proinflammatory cytokines from AT, which may activate endothelial cells and leukocytes within intrarenal microves- sels, affecting endothelial barrier function and eventually leading to irreversible tubular injury and nephron failure. Specifically, circulating levels of TNFa and IL-6 have been positively correlated with the incidence of CKD [6]. Also, experimental data in animal models have described the pathophysiological functions of many other adipokines and their potential contribution to developing CKD (shown in Fig. 1) [4].

\section{Leptin}

Not only obese, but also non-obese, patients with CKD present high serum leptin levels. Besides its effect on appetite and the contribution of hyperleptinemia to obesityassociated hypertension by overactivating the sympathetic nervous system [7], leptin can modulate different signalling pathways in the kidney, since glomerular endothelial and mesangial cells express abundant leptin receptors. Leptin induces an increased expression of profibrotic genes, such as TGF- $\beta 1$, and pro-inflammatory cytokines. TGF- $\beta 1$ can also bind its renal receptors, enhancing the expression of pro-fibrotic factors in a positive feedback loop. Furthermore, TGF- $\beta 1$ is a potent initiator of proliferation of renal mesangial cells. An increase in these molecules could thus cause thickening of the glomerular basement membrane, accumulation of the extracellular matrix, and glomerular mesangial hypertrophy, leading to, ultimately, glomerulosclerosis and proteinuria $[4,7]$.

\section{Adiponectin}

Adiponectin is primarily secreted by AT and is reported to have insulin-sensitizing, anti-inflammatory, and anti-apoptotic properties, probably through the activation of AMPK signalling $[4,7]$. Interestingly, adiponectin knockout mice develop microalbuminuria, kidney fibrosis, oxidative stress, inflammation, and podocyte dysfunction, whereas restoration of adiponectin levels rescues renal function [8].

Accordingly, a recent study has shown that a hybrid inhibitor blocking both cannabinoid 1 receptors and inducible nitric oxide synthase signalling can ameliorate obesity-related kidney injury partially through increasing adiponectin signalling in renal proximal tubule cells, which express both adiponectin and its receptors [9]. This concurs with the decreased levels of adiponectin in most patients with kidney disease $[4,7]$. Paradoxically, some studies show that patients with CKD and ESRD have high levels of this adipokine. The reason for this is still controversial, but it could be a compensatory mechanism, although a decrease in adiponectin sensitivity or a decrease in adiponectin clearance has also been suggested [10]. 


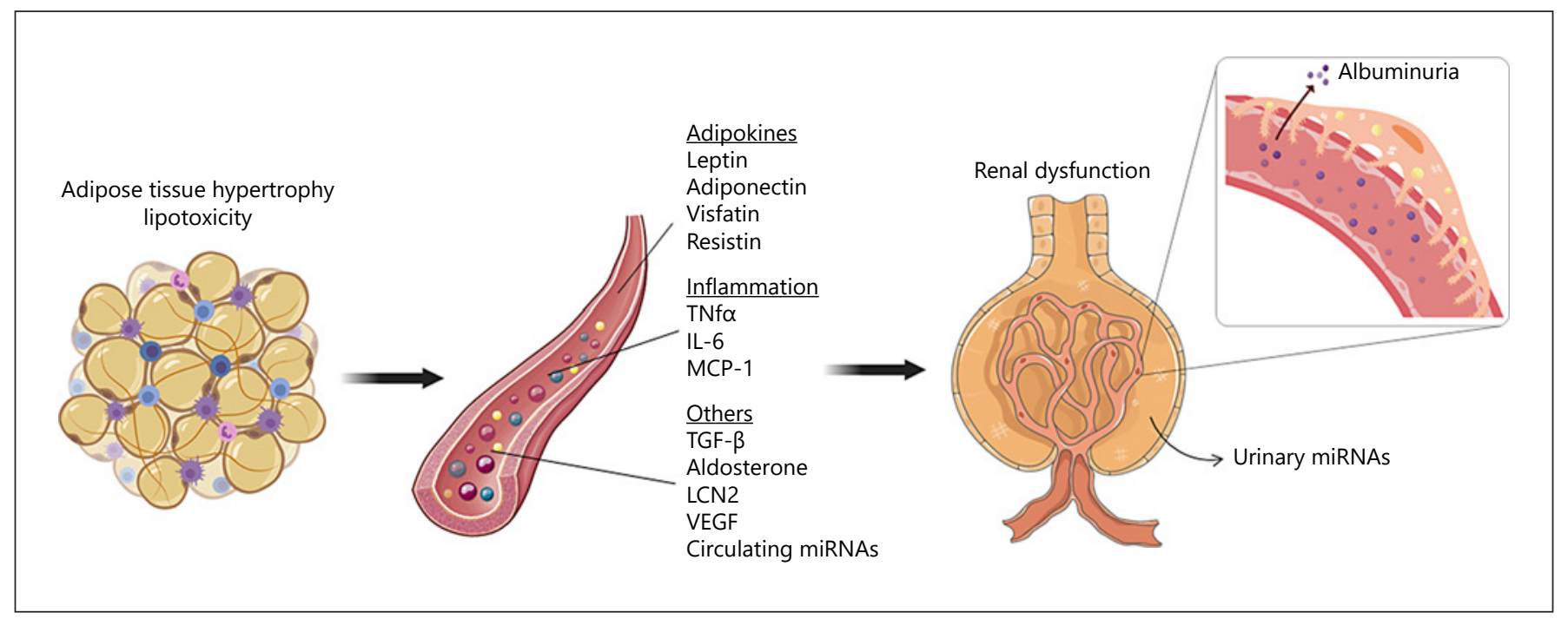

Fig. 1. Impaired AT functionality during obesity could affect normal renal function. Abnormal hypertrophy in the obese AT leads to lipotoxicity and the alteration of the adipocytokine secretion. Changes in the secretion of adipokines and inflammatory and fibrotic parameters, among others, may induce glomerulosclerosis, glomerular and podocyte hypertrophy, albuminuria and, therefore, chronic renal dysfunction. New potential biomarkers, such as microRNAs, might be helpful for early detection of kidney damage. AT, adipose tissue; miRNA, microRNA.

\section{Other Adipokines}

Visfatin and resistin display pro-inflammatory and atherogenic effects. Visfatin stimulates the expression of TGF- $\beta 1$, PAI-1, and type I collagen, having an important pro-fibrotic role, whereas resistin stimulates the production of ICAM-I and VCAM-I and promotes the activation of the sympathetic renal system. Levels of these adipokines are markedly elevated in obesity and CKD and correlate with pro-inflammatory parameters and decreased glomerular filtration rate (GFR) [7].

Besides the overactivation of the renin-angiotensinaldosterone system (RAAS) occurring during obesity, $\mathrm{AT}$ also produces components of the RAAS. Adipocytespecific deletion of angiotensinogen in mice reduces the plasma levels sufficiently to decrease systolic blood pressure. Also, adipocytes synthesize and secrete aldosterone, this being increased in obese animals [11]. Overactivated RAAS, alongside glomerulomegaly and dysregulation of sodium/glucose reabsorption, usually culminates in glomerular hypertension and hyperfiltration [2].

The pro-inflammatory adipokine lipocalin 2 (LCN2), also called neutrophil gelatinase-associated lipocalin, is considered a functional biomarker for acute kidney disease and CKD. LCN2 is known for its role in the innate immune response through its binding to siderophores derived from a bacterial infection. However, it can be secreted not only by neutrophils but also by other tissues such as the liver, lungs, or kidneys. Increased serum and urine levels of LCN2 have been reported in kidney injury, due to an augmented LCN2 expression in the renal distal tubule and impaired proximal tubule reabsorption [12]. In addition, AT is considered an important source for this adipokine. LCN2 expression and secretion are induced by pro-inflammatory cytokines in cultured adipocytes, and LCN2 levels are increased in AT and serum of individuals with obesity and obese mice $[12,13]$.

AT can also produce angiogenic factors such as VEGF. Increased levels of VEGF could induce the de novo formation of largely defective glomerular capillaries within the kidney, contributing to the glomerular hypertrophy characteristic of ORG [2].

\section{Management of Obesity-Associated Kidney Disease}

Pharmacological strategies for the treatment of obesity-linked CKD include RAAS blockers as well as diuretics and statins, the latter for the control of dyslipidemia, if present. Some authors bet on potential future drugs such as PPAR $\gamma$ synthetic agonists, as well as GLP-1 receptor agonists and SGLT2 inhibitors, classically used to amelio- 
rate glucose control, which have been recently shown to additionally improve kidney outcomes in patients with T2D [14]. This provides new opportunities for nephroprotection beyond RAAS blockade, maybe not only for patients with diabetic kidney disease but also for patients with ORG, given the similarities between both diseases. Clinicians otherwise promote weight loss to treat and prevent ORG [2], bariatric surgery (BS) being the most effective option to achieve the renal benefits of weight loss. Indeed, several authors have demonstrated the improvements in proteinuria, creatinine clearance, and GFR after BS [15]. However, more data regarding the impact of BS on renal disease are required to better characterise which patients could benefit most from this therapy.

BS exerts a renoprotective effect by reducing podocyte dedifferentiation and maintaining the correct glomerular structure, through the decreased expression of pro-inflammatory, pro-fibrotic, and stress signals in the renal cortex ([16] and references therein). Moreover, a recent study from our research group [17] indicates changes in adipocytokine secretion pattern as partially responsible for this improvement, as previously suggested [15].

\section{MicroRNAs in Obesity-Associated Kidney Disease: \\ Potential New Biomarkers}

BS interventions are commonly used when renal function is already compromised, since current non-invasive diagnosis of CKD relies on albuminuria and eGFR. Therefore, alternative strategies are necessary to identify at-risk patients in order to treat obesity-related nephropathy at an early stage.

MicroRNAs (miRNAs) are small non-coding RNA molecules now recognized as novel adipokines, considering that AT constitutes an important source for circulating miRNAs, which may modulate gene expression in distant organs [18]. In obesity, the adipocyte-derived miRNA pattern is altered, with some of these miRNAs targeting inflammatory and pro-fibrotic signalling pathways (reviewed in [13] and references therein). Therefore, altered miRNAs may ultimately contribute to obesity-associated renal dysfunction [18] and emerge as promising markers for the early diagnosis of CKD. Nevertheless, the study of miRNAs in the field of kidney disease is scarce and has been rather focused on urinary miRNAs, especially those encapsulated inside urinary extracellular vesicles. In fact, studies regarding the role of these miRNAs as potential biomarkers of ORG are still lacking.

\section{Conclusion}

Obesity and CKD often go hand in hand. However, the mechanisms of the detrimental effect of obesity on the kidneys remain elusive. Impaired AT functionality, lipotoxicity, dysregulation of AT secretion profile, and inflammation may affect renal function during obesity. Even though current diagnostic tools are being improved, the lack of early-onset biomarkers is evident. Within this framework, BS is central to treat obesity-related CKD, and the study of miRNAs opens new avenues in the discovery of biomarkers for the establishment and progression of this disease.

\section{Statement of Ethics}

The paper is exempt from ethical committee approval.

\section{Conflict of Interest Statement}

The authors have no conflicts of interest to declare.

\section{Funding Sources}

Research conducted for this publication was supported by Ministerio de Economía y Competitividad de España (BFU201678951-R, BFU2017-90578-REDT) and Comunidad de Madrid (Spain) (B2017/BMD-3684) to GMG and by Universidad Rey Juan Carlos, Madrid, Spain (Ayudas Puente, 2019) to RVB.

\section{Author Contributions}

M.M.T., R.V.B., and G.M.G. drafted and revised the paper. All authors approved the final version of the manuscript.

\footnotetext{
References

1 Ejerblad E, Fored CM, Lindblad P, Fryzek J, McLaughlin JK, Nyrén O. Obesity and risk for chronic renal failure. J Am Soc Nephrol. 2006; 17(6):1695-702.

2 D'Agati VD, Chagnac A, de Vries AP, Levi M, Porrini E, Herman-Edelstein M, et al. Obesity-related glomerulopathy: clinical and pathologic characteristics and pathogenesis. Nat Rev Nephrol. 2016;12(8):453-71.

3 Hashimoto Y, Tanaka M, Okada H, Senmaru T, Hamaguchi M, Asano M, et al. Metabolically healthy obesity and risk of incident CKD. Clin J Am Soc Nephrol. 2015;10(4): 578-83.
}

Martin-Taboada/Vila-Bedmar/ Medina-Gómez 
4 Zhu Q, Scherer PE. Immunologic and endocrine functions of adipose tissue: implications for kidney disease. Nat Rev Nephrol. 2018; 14(2):105-20

5 Martinez-Garcia C, Izquierdo-Lahuerta A, Vivas Y, Velasco I, Yeo TK, Chen S, et al. Renal lipotoxicity-associated inflammation and insulin resistance affects actin cytoskeleton organization in podocytes. PLoS One. 2015; 10(11): 0142291.

6 Lee BT, Ahmed FA, Hamm LL, Teran FJ, Chen CS, Liu Y, et al. Association of C-reactive protein, tumor necrosis factor-alpha, and interleukin-6 with chronic kidney disease. BMC Nephrol. 2015;16:77.

7 Briffa JF, McAinch AJ, Poronnik P, Hryciw $\mathrm{DH}$. Adipokines as a link between obesity and chronic kidney disease. Am J Physiol Renal Physiol. 2013;305(12):F1629-36.

8 Ohashi K, Iwatani H, Kihara S, Nakagawa Y, Komura N, Fujita K, et al. Exacerbation of albuminuria and renal fibrosis in subtotal renal ablation model of adiponectin-knockout mice. Arterioscler Thromb Vasc Biol. 2007; 27(9):1910-7.
9 Udi S, Hinden L, Ahmad M, Drori A, Iyer MR, Cinar R, et al. Dual inhibition of cannabinoid CB1 receptor and inducible NOS attenuates obesity-induced chronic kidney disease. Br J Pharmacol. 2020;177(1):110-27.

10 Navarro-Diaz M, Serra A, Lopez D, Granada M, Bayes B, Romero R. Obesity, inflammation, and kidney disease. Kidney Int Suppl. 2008(111):S15-8.

11 Briones AM, Nguyen Dinh Cat A, Callera GE, Yogi A, Burger D, He Y, et al. Adipocytes produce aldosterone through calcineurin-dependent signaling pathways: implications in diabetes mellitus-associated obesity and vascular dysfunction. Hypertension. 2012;59(5):106978.

12 Abella V, Scotece M, Conde J, Gómez R, Lois A, Pino J, et al. The potential of lipocalin-2/ NGAL as biomarker for inflammatory and metabolic diseases. Biomarkers. 2015;20(8): $565-71$.

13 Lee MW, Lee M, Oh KJ. Adipose tissue-derived signatures for obesity and type 2 diabetes: adipokines, batokines and microRNAs. J Clin Med. 2019;8(6):854
14 Williams DM, Nawaz A, Evans M. Renal outcomes in type 2 diabetes: a review of cardiovascular and renal outcome trials. Diabetes Ther. 2020;11(2):369-86.

15 Martin WP, Docherty NG, Le Roux CW. Impact of bariatric surgery on cardiovascular and renal complications of diabetes: a focus on clinical outcomes and putative mechanisms. Expert Rev Endocrinol Metab. 2018; 13(5):251-62.

16 Nair M, Martin WP, Zhernovkov V, Elliott JA, Fearon N, Eckhardt H, et al. Characterization of the renal cortical transcriptome following Roux-en-Y gastric bypass surgery in experimental diabetic kidney disease. BMJ Open Diabetes Res Care. 2020;8(1):e001113.

17 Morales E, Porrini E, Martin-Taboada M, Lima SL, Vila-Bedmar R, González de Pablos I, et al. Renoprotective role of bariatric surgery in patients with established CKD. Clin Kidney J. 2020.

18 Mori MA, Ludwig RG, Garcia-Martin R Brandão BB, Kahn CR. Extracellular miRNAs: from biomarkers to mediators of physiology and disease. Cell Metab. 2019;30(4): 656-73. 